How to reference this article

Campagnaro, M. (2017). Eredità del Novecento. II lascito munariano alla letteratura per I'infanzia. Italica Wratislaviensia, 8 (2), 69-85.

DOI: http://dx.doi.org/10.15804/IW.2017.08.18

Marnie Campagnaro

Università di Padova

marnie.campagnaro@unipd.it

\title{
EREDITÀ DEL NOVECENTO. IL LASCITO MUNARIANO ALLA LETTERATURA PER L'INFANZIA
}

\section{A TWENTIETH-CENTURY LEGACY: BRUNO MUNARI'S GIFT TO CHILDREN'S LITERATURE}

\begin{abstract}
Bruno Munari left an important legacy to both Italian and international children's literatures. The hallmarks of this legacy are traditionally considered to be his picture books and their unique visual narratives, illustrations, and tactile surfaces. This essay, however, takes a different approach to interpreting his work. It uses a selection of Munari's picture books to demonstrate how the extent of his legacy is far more wide-reaching, depicting it as a crossroads that links various features of his long career as an artist and designer. These features include the extraordinary nature of everyday life, faith in the visual, and the challenges laid down by limits, all of which are also fundamental to contemporary children's literature.
\end{abstract}

Keywords: Bruno Munari, picture books, childhood, visual literacy, Twentieth Century 


\section{INTRODUZIONE}

Tn una recente intervista, Rossellina Archinto, fondatrice della storica casa editrice Emme Edizioni, figura vitale dell'editoria italiana per ragazzi della seconda metà del Novecento, afferma, in qualità di editore di alcune sue opere, che Bruno Munari, pur avendo fatto della grafica magnifica, dei libri straordinari, pur essendo stato un meraviglioso comunicatore, con idee geniali, che sapeva far divertire moltissimo i bambini, non può essere propriamente considerato un autore di libri per bambini (Farina, 2013, p. 71).

L'affermazione dell'editora milanese appare a prima vista una contraddizione, apparentemente inconciliabile con la storia autoriale di questa figura, dal momento che Munari ha estensivamente scritto e illustrato opere di letteratura per l'infanzia. Ha pubblicato con numerose case editrici quali Mondadori, Muggiani, Emme Edizioni, Danese, Einaudi, Zanichelli, Corraini. Per i tipi della Emme Edizioni, ha pubblicato albi innovati quali Nella nebbia di Milano (1968), Da lontano era un'isola (1971), Un fiore con amore (1973).

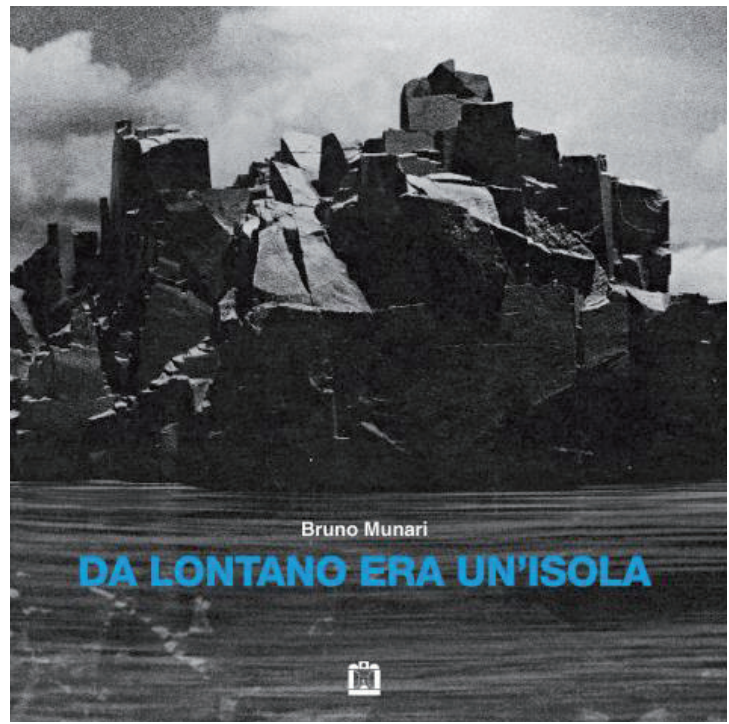

Fig. 1. B. Munari, Da lontano era un 'isola, Mantova, Corraini, 2008 (copertina) 
Per Einaudi, è bene ricordarlo, oltre ai celeberrimi lavori di innovazione grafica (basta citare il suo lavoro per collane significative quali "Nuova Universale Einaudi", "Piccola Biblioteca Einaudi", "Nuovo Politecnico"), Munari ha dato forma e colore alle celebri storie di Gianni Rodari, Filastrocche in cielo e in terra (1960), Favole al telefono (1962), Il pianeta degli alberi di Natale (1962), Il libro degli errori (1964), La torta in cielo (1966), ha progettato e diretto un'innovativa collana editoriale "Tantibambini" (1972-1978), nella quale chiamò a raccolta alcuni fra i più rilevanti scrittori, poeti, grafici, illustratori ed artisti del tempo. Dei sessantasei albi illustrati che compongono la collana, dieci sono il frutto autoriale di Munari (quattro sono stati pubblicati sotto l'ironico pseudonimo di E. Poi).

Questi brevi cenni paiono, dunque, non dare credito alle parole di Archinto. E, invece, va dato merito a questa signora dell'editoria italiana di aver saputo intercettare, nella sua affermazione, uno dei tratti più caratterizzanti la figura poliedrica di Munari, ovvero la sua inafferrabilità, sia perché Munari "non si lascia definire, né incasellare in alcuna definizione" (Meneguzzo, 1993, p. 2) sia perché i numerosissimi progetti e le tante attività condotte in campi diversi (arte, grafica, design, editoria, fotografia, educazione, ecc.), portati avanti nel corso di oltre settant'anni di impegno professionale, godono di una sostanziale "indivisibilità" (p. 51). Non a caso Alessandro Mendini nella presentazione di un volume del 1979 dedicato all'artista milanese, considerato uno dei designer del XX secolo più importanti al mondo, dichiara che "È difficile dire che cosa è Munari. Meglio aggirare l'ostacolo e dire cosa egli «non è». Munari, allora, non è un designer, non un grafico, non un cartellonista, non un vetrinista, non uno stilista, non un saggista, non un insegnante, non uno scultore, non un fotografo, non un regista, non un poeta, non un semiologo, non un bambino, non un adulto, non un vecchio, non un giovane" (p. 55).

Eppure, Munari, da "non autore" di libri per bambini, ha lasciato un'importante eredità alla letteratura per l'infanzia di ieri e di oggi. Questo lascito non è legato ad uno stile narrativo o illustrativo, che in Munari, praticante inesausto del "nomadismo culturale" (Meneguzzo, 1993, p. 57), non si può dare, né tanto meno ai contenuti o alle forme grafico- 
tipografiche dei suoi libri, anche se è indubbio che alcune sue opere hanno contribuito a svecchiare, e non poco, la letteratura per l'infanzia italiana. Si pensi, a titolo esemplificativo, alla capacità visionaria manifestata nella progettazione della serie di libri per la Mondadori nel 1945, oggi ristampata dalla Corraini, o all'albo Nella notte buia del 1956, considerato una delle opere caposaldo nella storia dell'albo illustrato.

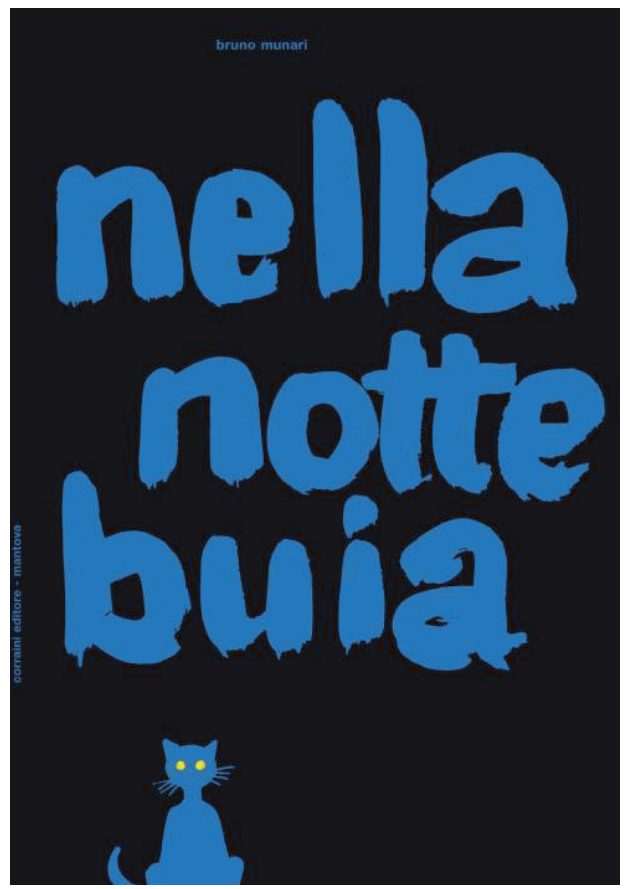

Fig. 2. B. Munari, Nella notte buia, Mantova, Corraini, 2017 (copertina)

A parere di chi scrive il lascito di Munari alla letteratura per l'infanzia, al netto delle narrazioni sentimentali sul personaggio, si coglie ad altro livello, in uno specialissimo crocevia al quale fanno capo distinti elementi della sua lunga attività artistica: la straordinarietà del quotidiano, la fiducia nel visivo, la sfida del limite. È di questi elementi, dunque, che ci vogliamo occupare. 


\section{LA STRAORDINARIETÀ DEL QUOTIDIANO}

Animato da genialità creativa e da capacità di sperimentazione inesauribili, il lavoro di Bruno Munari contiene alcuni elementi ricorrenti: lo studio rigoroso della materia e dei materiali, la ricerca della semplicità e dell'essenzialità, la leggerezza del gesto ludico, spiazzante e demistificante, il coinvolgimento attivo del fruitore, in particolare del bambino, e una buona dose di "ironia socratica" (Meneguzzo, 1993, p. 25). La serietà garbatamente canzonatoria, particolarmente evidente in alcune sorprendenti creazioni artistiche, come nelle "forchette parlanti" o nelle "macchine inutili", mettono in evidenza il senso di divertissement del suo gesto creativo (Fiz, 2000, p. 24). Fra gli aspetti meno analizzati, tuttavia, vi è un elemento, che, ai fini della nostra analisi, merita di essere evidenziato: si tratta per l'appunto del ruolo della creatività perimetrata sul reale.

Munari ha tessuto un rapporto peculiare con la dimensione della quotidianità: essa rappresenta il bacino immaginario da cui le sue idee creative prendono spunto e si riverberano. Come ci ricorda lo storico dell'arte e critico Filiberto Menna, l'artista milanese non ha mai avuto alcun desiderio di destare stupori collettivi o di frequentare mondi sospesi fantastici ed irreali. Ciò che gli premeva era la possibilità di creare piccoli mondi favolosi e microcosmi incantati negli ambienti di tutti i giorni (Menna, 1966, p. 15): creazioni "straordinarie", per l'appunto, per un uso quotidiano e domestico. Le ambientazioni, che fanno da cornice alle avventure narrative in certi libri, sono "fantastiche" non perché ritraggono luoghi irreali, immaginari o fittizi usciti dalla fantasia di Munari, ma perché scagliano il protagonista in spazi a margine dell'ordinaria routine: ad esempio, sono l'inusitata scelta del tempo o delle condizioni metereologiche che rendono singolare e fantastica l'avventura (Campagnaro, Goga, 2014, p. 80). Le forme bizzarre, eccentriche e ilari delle sue ambientazioni nascono dalla scelta di proporre momenti peculiari della quotidianità: una nebbia, talmente fitta da celare spazi speciali della città (Nella nebbia di Milano, 1968), un traffico cittadino, intasato a tal punto da essere più pericoloso dell'attraversamento di un bosco (Cappuccetto Giallo, 1972), una neve, così abbondante da riuscire a ricoprire ogni cosa (Cappuccetto Bianco, 1981) 


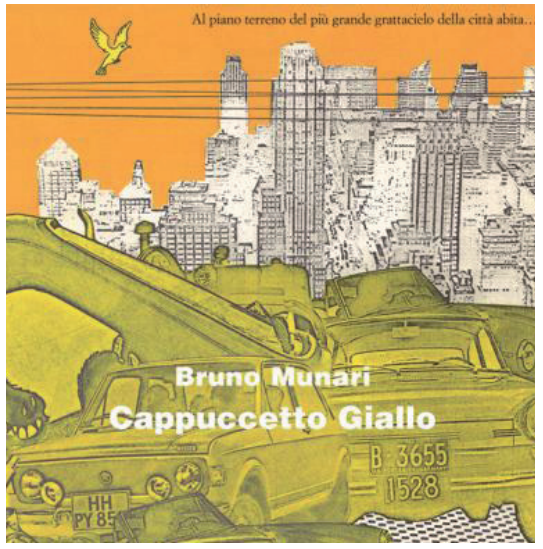

Fig. 3. B. Munari, Cappuccetto Giallo, Mantova, Corraini, 2014 (copertina)

\section{Bruno Munari \\ Cappuccetto Bianco}

Fig. 4. B. Munari, Cappuccetto Bianco, Mantova, Corraini, 2017 (copertina)

Per Munari, la dimensione dell'inaspettato, dell'imprevisto, del favoloso sta nella quotidianità di tutti i giorni, l'ignoto è "dentro a ciò che conosciamo, le declinazioni del reale che non abbiamo l'abitudine a guardare" (Tassinari, 1996/2006, p. 10). Per Munari, la creatività non è un'invenzione del nuovo, ma la rivelazione delle zone inesplorate dell'esistente, esplorazioni che alla fin fine sono capaci di stupire di più 
e di essere più disarmanti (p. 10) perché offrono la possibilità di cogliere l'universo nelle piccole infinite manifestazioni di ogni giorno.

\section{LA FIDUCIA NEL VISIVO}

Il desiderio e la premura di voler donare al figlio Alberto, nato nel 1940, libri più adatti alla curiosità e all'ingegno infantile spinge Munari nel 1945 a progettare per la Mondadori una serie di dieci libri (ne saranno tuttavia pubblicati solo 7). Sono opere destinate a rimettere in discussione l'idea stessa di libro per bambini perché Munari compie un gesto che, seppure silenzioso, appare, allora come oggi, rivoluzionario. Egli decide di "sfiduciare" la parola e di assegnare il primato all'immagine, perché quest'ultima, grazie alle sue autonome capacità comunicative, può permettersi di dialogare direttamente con i bambini (Maffei, 2008, p. 27).

Munari è stato uno straordinario sperimentatore della comunicazione visiva. Questo fervore gli ha consentito di testare le condizioni più adatte e performanti per offrire ad adulti e bambini, attraverso il linguaggio visuale, conoscenze e sollecitazioni estetiche.

L'opera Nella nebbia di Milano (1968) ne è un esempio felice.

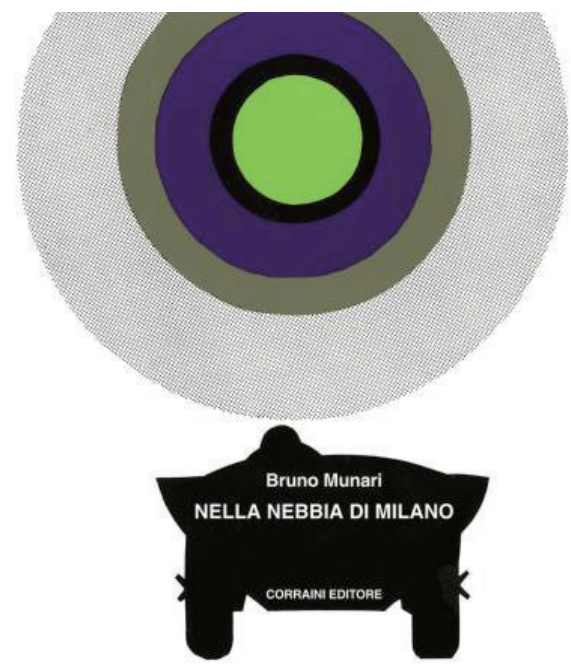

Fig. 5. B. Munari, Nella nebbia di Milano, Mantova, Corraini, 2008 (copertina) 
L'albo rappresenta lo straordinario omaggio di Munari alla sua città, Milano, il centro di tutta la sua attività artistica, una città che ha amato profondamente perché gli ha permesso di realizzare quanto più desiderava (Tanchis, 1986, p. 11). L'opera, tuttavia, è anche un'affettuosa reminiscenza del paesaggio della sua infanzia, trascorsa libera, all'aria aperta in un paesino della campagna veneta presso l'Adige, a Badia Polesine, in un'area rurale, dislocata a sud-ovest di Venezia, vicinissima a Rovigo e a Padova. La campagna rodigina degli anni Venti, fatta di campi sterminati, lunghi filari di alberi, acqua di fiume che scorre, vecchi mulini di legno che sembrano costruiti da Robinson Crusoe, ha forgiato il patrimonio immaginativo dell'artista milanese (pp. 6-8), ma è stata indubbiamente anche un'utilissima scuola a cielo aperto per imparare ad osservare. In questo suo errare ludico a stretto contatto con la natura, fatto di silenzi, esplorazioni e scoperte inattese, Munari comprende quanto la natura possa essere varia, flessibile, duttile, capace di rifuggire "da rigidità concettuali e progettuali" (Meneguzzo, 1993, p. 6): dietro l'infinita variabilità delle forme e delle strutture, ci sono "elementi semplici, definiti e pochi. Triangolo equilatero, quadrato e cerchio risultano essere la base di ogni struttura, quindi di ogni costruzione e di ogni oggetto" (p. 6). Anche la genesi di Nella nebbia di Milano può essere ricondotta all'innovativa e variegata intesa fra questi elementi. L'albo è costruito sul singolare dialogo grafico-visivo di tre sezioni, ciascuna delle quali fotografa una situazione particolare della vita di un bambino a Milano. La prima sezione descrive la vita urbana della città immersa nel traffico e avvolta nella nebbia, la seconda sezione racconta il turbinio di personaggi, forme e colori incontrati durante una visita al circo, si ipotizza, appena fuori il centro cittadino, la terza e ultima sezione illustra la passeggiata di ritorno del protagonista a casa attraverso il verde e la natura di un parco limitrofo. Munari costruisce i tre paesaggi, declinando, secondo una felice similitudine visiva, le forme geometriche primarie: triangolo equilatero, quadrato e cerchio. Egli costruisce il paesaggio urbano, tipicamente caratterizzato da una forte impronta architettonica, mantenendo intatta la forma delle figure geometriche e riproducendo i vari elementi che lo animano (veicoli, semafori, segnali stradali, fontane, siluette delle persone, ecc.), sommando e/o moltipli- 
cando, secondo dimensioni differenziate, tali elementi. Per la sezione successiva, ovvero il paesaggio del circo, molto più morbido e imprevedibile, egli utilizza le stesse forme geometriche ma, in questo caso, per dare vita ai personaggi umani, agli animali del circo e ai loro strumenti ginnico-ludici, Munari le comprime, le allunga, le deforma, ne fa un uso iperboleggiato, quasi a voler ammiccare al favoloso e inafferrabile mondo dell'arte. La sintesi fra rigidità geometrica del paesaggio urbano e duttilità dell'universo artistico rappresentata dal circo ritorna visivamente nella sezione finale: le forme degli alberi, dei rami, dei cespugli, delle foglie, che il protagonista incontra, passeggiando nel bosco, altro non sono che il prodotto delle trasformazioni subite da queste forme organiche nel processo di adattamento alle contingenze dell'ambiente, della vita (Meneguzzo, 1993, p. 7).

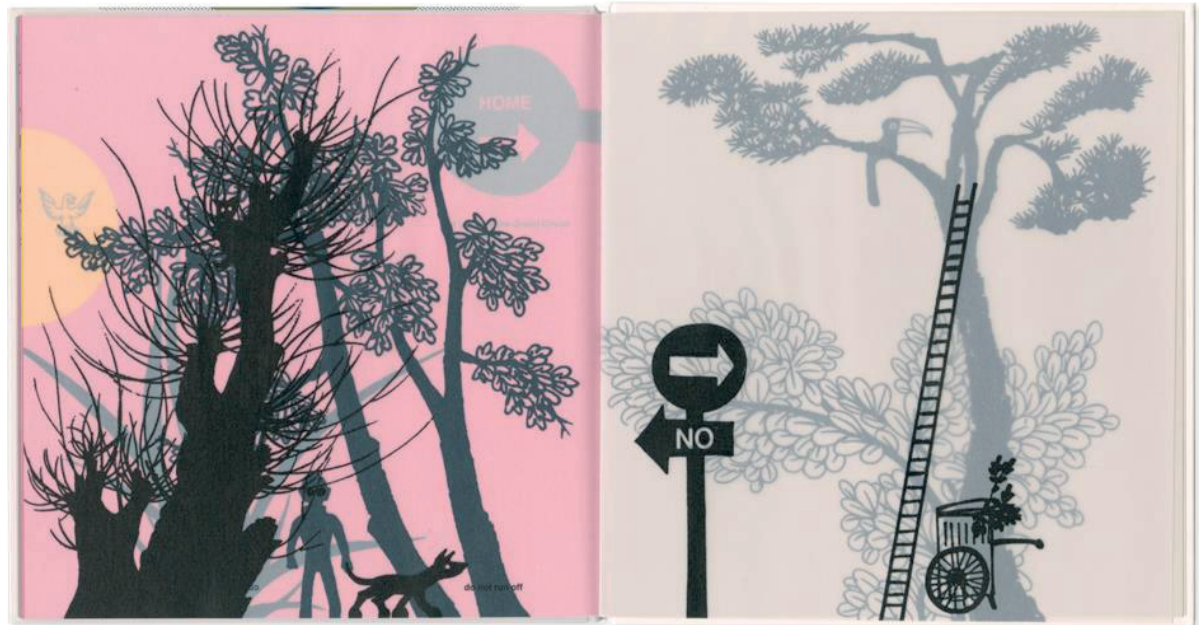

Fig. 6. B. Munari, Nella nebbia di Milano, Mantova, Corraini, 2008 (pagine interne)

La formazione giovanile, "dentro la campagna", ha permesso a $\mathrm{Mu}-$ nari di imparare ad osservare la natura, attivare tutti i ricettori sensoriali, non solo quelli notoriamente collegati ai cinque sensi ma anche quelli meno manifesti, eppure altrettanto importanti, quali, ad esempio, il senso del ritmo, il senso del peso, il senso dell'equilibrio, che immancabilmente troviamo in tante sue opere artistiche. Fra essi, la percezione visi- 
va, sviluppata ai tempi dei giochi all'aria aperta durante la sua infanzia, ha creato una riserva enorme di suggestioni che lo accompagnerà per tutta la vita e che gli permetterà di osservare la realtà non solo con occhi diversi ma anche secondo logiche ai più sconosciute.

\section{LA SFIDA DEL LIMITE}

Già all'inizio degli anni Settanta, oltre all'intensa attività editoriale, si consolida sempre più in Munari, sino a diventare centrale nell'ultima fase della sua intensa carriera, l'attenzione per la progettazione didattica. Munari è avanti nel tempo: la lunga onda della contestazione giovanile è ancora pulsante, "tutti parlano dei giovani, Munari [invece] parla dei bambini” (Meneguzzo, 1993, p. 56). Sono gli anni in cui Munari, fra le molte fortunate realizzazioni, lavorerà anche alla messa a punto dei primi innovativi laboratori didattici dedicati ai bambini*. L'osservazione della natura, l'attenzione all'arte, l'educazione al visivo e all'estetica, il confronto con la quotidianità, l'idea di funzionalità, il meccanismo del gioco, lo stupore dell'infanzia, l'ironia garbata e il divertimento puro hanno profondamente nutrito moltissime opere di Munari, fra cui i laboratori. Tuttavia, questi elementi non sarebbero stati sufficienti a liberare i risultati più imprevedibili e fecondi del suo lavoro, se non si fossero armonicamente amalgamati con la logica scientifica, il ragionamento razionale e l'applicazione rigorosissima di un metodo messo a punto dall'artista milanese (Ballo, 1965, p. 70). Questo metodo si avvale di una particolare potenzialità euristica, quella cioè legata all'esperienza del limite.

Il limite è un confine, una linea terminale o divisoria, che separa due territori, due stati, due situazioni, che delimita i confini di un podere, di una città, di un mondo. Esso si configura anche come confine ideale, il livello massimo, al disopra o al disotto del quale si verifica normal-

Il primo è progettato da Munari nel 1977 presso la Pinacoteca di Brera a Milano. Due anni dopo, Munari organizza il secondo laboratorio presso il Museo Internazionale delle Ceramiche di Faenza. L'esperienza sarà così feconda che i suoi laboratori si moltiplicano ed iniziano a diffondersi non solo in Italia, ma anche all'estero. 
mente un determinato fenomeno. Il limite impone un contenimento, una riduzione, un restringimento che impedisce di andare più in là (Rigotti, 2004, p. 41). Eppure, al contempo, oltre che a restringere e a contenere, il limite sa essere anche oggetto tentatore, capace di infiammare l'immaginazione e di spingerla verso un'ulteriorità, uno spazio che, prima dell'esplorazione, non è dato conoscere. Le narrazioni visive progettate da Munari nei suoi libri per bambini utilizzano questo meccanismo e sovente sottopongono il lettore al confronto con il vincolo di liminalità. Nell'attività di sperimentazione di Munari, 1'esperienza del limite ha giocato un ruolo fondamentale. Come ha ben sintetizzato Marco Meneguzzo, essa, di fatto, rappresenta "l'esatta applicazione di un metodo" (2000, p. 23). Ma in cosa consiste l'applicazione di questo metodo?

Per Munari, riflettere intorno al concetto di limite ha primariamente significato riflettere sulla necessità di frequentare luoghi marginali, poco battuti, di esplorarli per ricavare preziosi dati di realtà. Nel corso della sua storia, il designer milanese è stato capace di percorrere, di sostare e, spesso, di scavare nicchie profonde proprio sul confine fra due territori. Si pensi, ad esempio, ai suoi lavori a margine fra arte e design industriale o alla frequentazione assidua di "luoghi «laterali» come studi d'architettura, grandi negozi, studi fotografici - come quello americano di Leo Lionni - o [...], musei” (Meneguzzo, 1993, p. 53). La sua marginalità ha fatto scuola, e ciò che un tempo era considerato territorio inesplorato, poco o per nulla interessante, grazie al suo lavoro, si è trasformato in "una specie di «marca», un luogo di confine ove effettuare incursioni proficue, dal punto di vista della creatività" (p. 57). Dal confronto con il limite, con uno stato, dunque, di privazione e di limitatezza, Munari ha tratto le idee e le risorse per accendere il desiderio di conoscenza, tanto nell'adulto quanto nel bambino. Per il nostro "non autore", e qui veniamo alla nostra domanda, l'applicazione di questo metodo si è sostanziata nell' "andare a vedere fino a che punto un oggetto, un'idea, una persona possono arrivare senza esserne snaturate" (p. 23). Nel caso dei libri per bambini, si tratta di indagare le molteplici possibilità attraverso cui un'esperienza narrativa può essere consegnata ad un giovane lettore. È per l'appunto il caso dei suoi Libri illeggibili, la cui prima esperienza del 1949 ha dato l'input a numerose successive sperimentazioni che si 
sono protratte sino agli anni Novanta, o dei Prelibri (1981), una singolare esperienza narrativa tattile-sensoriale per bambini piccolissimi.
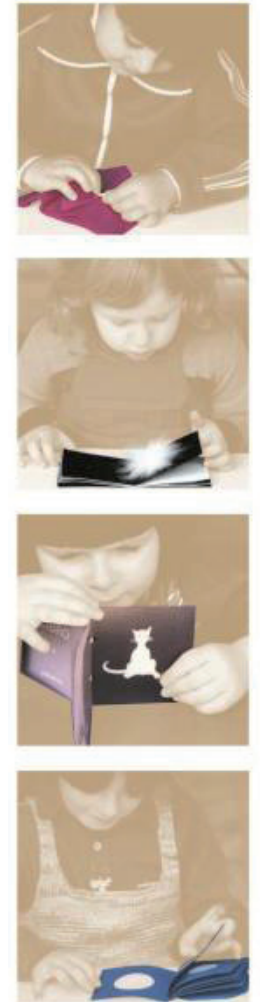
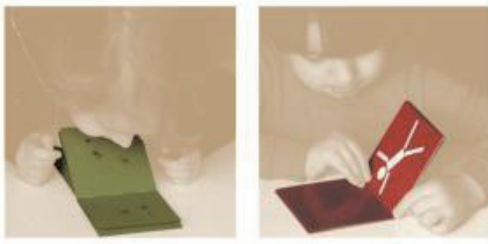

BRUNO MUNARI

PREBOOKS VORBÜCHER PRELIVRES

\section{| PRELIBRI}
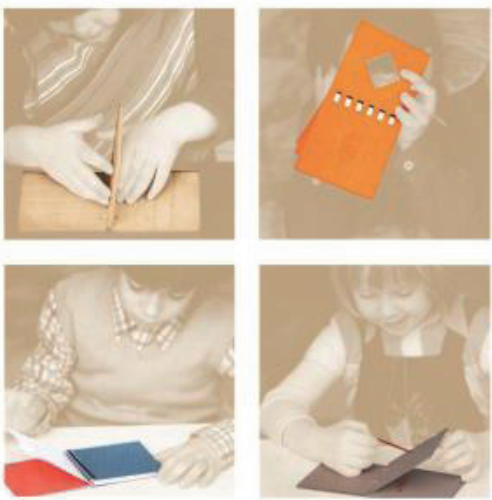

Fig. 8. B. Munari, I prelibri, Mantova, Corraini, 2016 (copertina)

La sfida del limite è il mezzo ideale per mettere in moto i pensieri dei propri lettori, per sollecitarli ad appropriarsi esteticamente di nuovi sguardi, di nuove prospettive sui fatti che stanno dentro, intorno o addirittura fuori i suoi libri. È il caso, ad esempio, dei già citati Libri illeggibili o dei Prelibri, ove "lo scopo di questa sperimentazione è stato quello di vedere se è possibile usare il materiale col quale si fa un libro (escluso il testo) come linguaggio visivo. Il problema quindi è: si può comunicare visivamente e tattilmente, solo con i mezzi editoriali di produzione di un libro? Ovvero: il libro come oggetto, indipendentemente 
dalle parole stampate, può comunicare qualcosa? E che cosa?" (B. Munari, 1981/2009, p. 217).

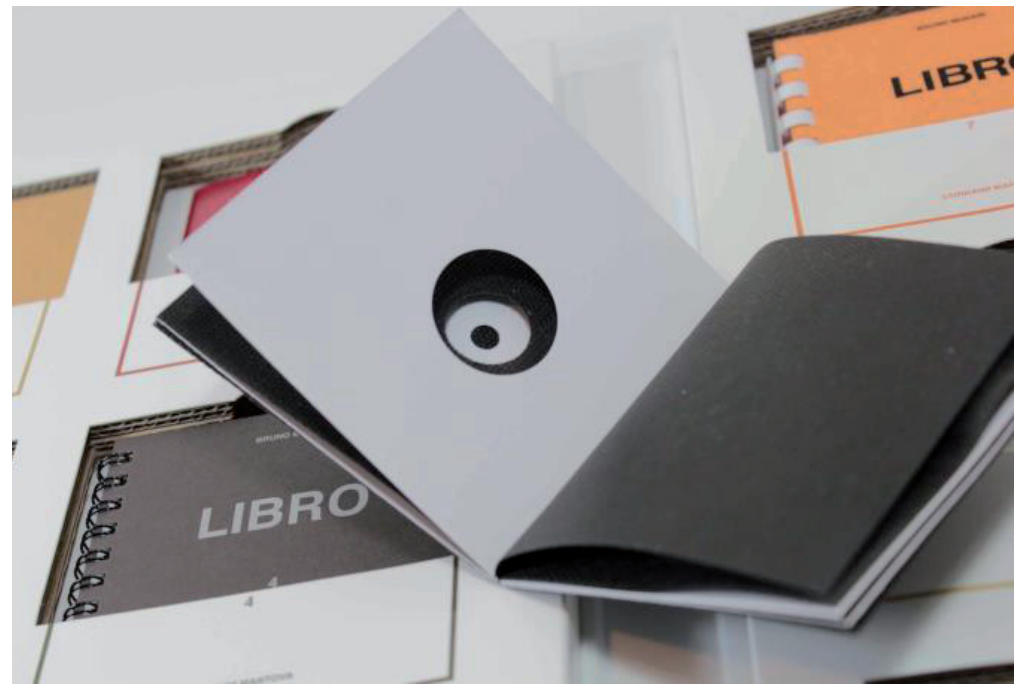

Fig. 9. B. Munari, Nella nebbia di Milano, Mantova, Corraini, 2016 (pagine interne, Libro n. 10)

L'azione di Munari offerta dall'esperienza del limite si è manifestata, giocando su attraversamenti, sconfinamenti, mescolanze di generi diversi (Campagnaro, 2016): letterari, visivi, artistici, culturali, educativi: "il mondo va guardato con la mente sgombra di pregiudizi e con un'attitudine costruttiva nei confronti delle cose" (Meneguzzo, 1996, p. 3). Il punto di partenza concettuale e il metodo di analisi sono tutt'altro che ingenui e vengono da lui applicati anche nei diversi universi educativi, sin dalla tenera età, "perché quello che si impara nei primi anni di vita resta come regola fissa per sempre" (B. Munari, 1981/2009, p. 228). L'esperienza del limite si rivela una risorsa autorevole per scoprire e indagare territori inesplorati anche con i bambini, dal momento che essa è legata al modo straordinario di vedere e di fare le cose, un modo fuori dal comune. Rispetto ad un uso standard, medio, e spesso mediocre del libro, Munari sperimenta e traccia una via per sfruttare le molteplici possibilità offerte dal mezzo: artists'books, book-objects, book-games, 
wordless books, material-books, cut-out books, book-sculptures, ecc. (Beckett, 2012).

Nelle sue inesauribili declinazioni, il libro per bambini diventa un alleato preziosissimo, una palestra indispensabile per formare il pensiero critico e il senso estetico delle giovanissime generazioni.

\section{CONCLUSIONI}

A voler cercare informazioni sul lascito munariano nella storia della letteratura per l'infanzia, si trovano solo pochi cenni. Egli, in genere, è ricordato per essere un interprete della gratuità del segno, dell'inutilità della parola, del gioco dell'arte, per essere stato capace di proporre soluzioni tipograficamente geniali: "libri fatti con materiali diversi dalla carta, [...], rivisitazioni di fiabe tradizionali o di riconoscibilissimi motivi popolari, proposte di «percorsi vegetali», insomma una pluralità di idee, di stimoli, di provocazioni in grado di aprire molti percorsi agli illustratori-scrittori delle generazioni successive" (Boero, De Luca, 1995/2007, p. 303). La storia dell'illustrazione italiana gli dedica poco meno di dieci righe (Pallottino, 2010). Più ricche e approfondite sono le analisi condotte sui temi del visivo (Beckett, 2002, Terrusi, 2012), sull'evoluzione grafico-tipografica dei suoi albi illustrati (Beckett, 2012) o sull'innovativa esperienza della collana editoriale "Tantibambini”" (Franchi, 2014).

Si tratta, tuttavia, di analisi che si sono soffermate più sulla descrizione dei contenuti delle sue opere, sulla diversità di utilizzo dei materiali, sull'innovazione del lay-out progettuale-grafico-tipografico dei suoi libri-oggetto, e meno sugli elementi costitutivi e sulle finalità della sua poetica, sul personalissimo dialogo storico-culturale che Munari, nel corso del secondo Novecento, ha consegnato alle giovani generazioni attraverso i suoi libri. Forse, perché la sua, che è stata una rivoluzione garbata e silenziosa, non ha fatto molto rumore, al di là della risonanza dei suoi laboratori didattici.

Eppure, nel leggere e nel guardare le creazioni di Munari si possono cogliere degli orientamenti di studio e ricerca sulla letteratura per l'infanzia che vanno ben oltre le coordinate di analisi sopra accennate. 
Innanzitutto, si segnala il tentativo di promuovere nei bambini l'interesse a cercare nuove vie per coltivare un'intima vocazione umana, ossia il desiderio di conoscere il mondo in cui si vive. Senza forzature, attraverso la pratica dilettevole del libero esercizio. Per Munari, indicare una nuova via "non significa per forza doverla seguire... In questo senso la componente ironica del suo lavoro - che tuttavia non è né fondamentale, né preponderante - non è mai feroce, ma sorridente, ed è solo un corollario, un artificio che fa accettare più facilmente lo stravolgimento delle regole, la discussione dei codici, l'accantonamento delle abitudini: l'ironia stempera la violenza di un'operazione la quale, se operata senza leggerezza, rischia di essere insopportabile, e, quindi, di venire rigettata" (Meneguzzo, 1996, p. 3).

Munari, inoltre, consegna ai suoi lettori l'importanza di guardare alla realtà con uno sguardo rinnovato e la possibilità di farlo attraverso nuove modalità, quali, ad esempio, la straordinarietà del quotidiano, la fiducia nel visivo e la sfida che si nasconde dietro un limite.

Sono modalità operative che Munari ha saputo intuire già negli anni Quaranta, con la prima produzione seriale per la Mondadori, ma che poi ha incessantemente coltivato nei successivi cinquant'anni. Esplorando empiricamente e con l'aiuto delle teorie educative sull'infanzia del tempo, in primis quelle sviluppate dall'epistemologo svizzero Jean Piaget, Munari comprese velocemente i danni devastanti che il crollo della curiosità, del libero interrogarsi sui fatti del mondo, l'appiattimento e il conformarsi a certe modalità del far educativo possono indurre nello sviluppo della personalità di un bambino (A. Munari, 2000). Mettere il bambino di fronte "alla vastità delle possibilità, alle diversità, alla non-norma" è stato, in fondo, il suo tentativo di dare una risposta a questa impellenza educativa. Lo ha fatto progettando libri singolari, che procedendo dall'osservazione del quotidiano, attraverso efficaci e "straordinarie" esemplificazioni, instillano nel bambino la fiducia di poter operare anche zigzagando per le vie del mondo. 


\section{BIBLIOGRAFIA}

\section{Bibliografia primaria}

Munari, B. (1956). Nella notte buia. Milano: Muggiani.

Munari, B. (1968). Nella nebbia di Milano. Milano: Emme Edizioni.

Munari, B. (1971). Da lontano era un 'isola. Milano: Emme Edizioni.

Munari, B. (1972). Cappuccetto Giallo. Torino: Giulio Einaudi.

Munari, B. (1973). Un fiore con amore. Milano: Emme Edizioni.

Munari, B. (1981). Cappuccetto Bianco. Milano: Muggiani.

Munari, B. (1981). I prelibri. Milano: Danese.

G. Rodari, Munari, B. (1960). Filastrocche in cielo e in terra. Torino: Giulio Einaudi.

G. Rodari, Munari, B. (1962). Il pianeta degli alberi di Natale. Torino: Giulio Einaudi.

G. Rodari, Munari, B. (1962). Favole al telefono. Torino: Giulio Einaudi.

G. Rodari, Munari, B. (1964). Il libro degli errori. Torino: Giulio Einaudi.

G. Rodari, Munari, B. (1966). La torta in cielo. Torino: Giulio Einaudi.

\section{Bibliografia secondaria}

Ballo, G. (1965). Designers italiani. Con Bruno Munari continua la galleria dei personaggi che hanno inciso sull'evoluzione del costume artistico italiano. Ideal Standard, 1-2, Reperibile su www.munart.org/doc/bruno-munari-g-ballo-ideal-standard-1965.pdf

Beckett, S. (2002). Recycling Red Riding Hood. New York-London: Routledge.

Beckett, S. (2012). Crossover Picturebooks: A Genre for All Ages. New York/ Oxon: Routledge.

Boero, P., De Luca, C. (1995/2007). La letteratura per l'infanzia. Roma-Bari: Laterza.

Campagnaro, M. (2016). The Function of Play in Bruno Munari's Children's Books. A Historical Overview. Ricerche di Pedagogia e Didattica Journal of Theories and Research in Education, 11 (3), 93-105.

Campagnaro, M., Goga, N. (2014). Bambini “in cammino" nella letteratura per l'infanzia. Forme e figure nei libri illustrati. In M. Campagnaro (a cura di), Le terre della fantasia. Leggere la letteratura per l'infanzia e l'adolescenza (pp. 73-98). Roma: Donzelli. 
Farina, L. (a cura di). (2013). La casa delle meraviglie. La Emme Edizioni di Rosellina Archinto. Milano: Topipittori.

Fiz, A. (2000). Bruno Munari tra spiazzamento e demistificazione. In A. Fiz (a cura di), Omaggio a Bruno Munari (pp. 15-21). Mazzotta: Milano.

Franchi, G. (2014). Le regole del gioco. I tantibambini di Bruno Munari. In S. Sola e P. Vassalli (a cura di), I nostri anni '70. Libri per ragazzi in Italia (pp. 94-99). Mantova: Corraini.

Maffei, G. (2008). Munari i libri. Mantova: Corraini, 2008.

Meneguzzo, M. (1993). Bruno Munari. Roma-Bari: Editori Laterza.

Meneguzzo, M. (1996). Ritratto dell'artista da artista. Catalogo mostra "Bruno Munari Opere 1930/1995. Bergamo: Galleria Fumagalli Bergamo, Edizioni Stefano Fumagalli. Reperibile su http://www.munart.org/doc/ bruno-munari-m-meneguzzo-fumagalli-1996.pdf

Menna, F. (1966). Bruno Munari o la coincidenza degli opposti. La botte e il violino. Repertorio illustrato di design e di disegno, (8), 11-18.

Munari, A. (2000). Munari: Piaget e Munari. In G.C. Bojani e D. Valli (a cura di), Munari. Arte come didattica. Atti del convegno di studi Faenza 17 aprile 1999, Museo Internazionale della Ceramica (pp. 17-22). Firenze: Centro Di.

Munari, B. (1981/2009). Da cosa nasce cosa. Roma-Bari: Laterza.

Pallottino, P. (2010). Storia dell'illustrazione italiana. Cinque secoli di immagini riprodotte. Firenze: Uscher/VoLo Publisher.

Rigotti, F. (2004). La filosofia delle piccole cose. Novara: Interlinea.

Tanchis, A. (1986). Bruno Munari. Milano: Idea Books Edizioni.

Tassinari, V. (1996/2006). Ma chi è Bruno Munari? Mantova: Corraini.

Terrusi, M. (2012). Albi illustrati. Leggere, guardare, nominare il mondo dei libri per l'infanzia. Roma: Carocci.

Riassunto: Bruno Munari ha lasciato un'importante eredità alla letteratura per l'infanzia, non solo a livello italiano, ma anche internazionale. In genere, si ritiene che questo lascito sia costituito dall'insieme delle sue singolari narrazioni visive e delle sue innovative soluzioni grafico-tipografiche. Il saggio, invece, mette in evidenza, anche attraverso la presentazione di alcuni albi illustrati scelti a titolo esemplificativo, un differente orientamento interpretativo per dimostrare che l'apporto di Munari è molto più vasto poiché rappresenta uno specialissimo crocevia che riannoda vari elementi della sua lunga attività artistica, fondamentali per la letteratura destinata all'infanzia: la straordinarietà del quotidiano, la fiducia nel visivo, la sfida del limite.

Parole chiave: Bruno Munari, albi illustrati, infanzia, alfabetizzazione visiva, Novecento 